\title{
О ПРИРОДЕ РАССЕЯНИЯ АКУСТИЧЕСКИХ ВОЛНОВЫХ ПАКЕТОВ ТОНКОСТЕННЫМИ УПРУГИМИ ЦИЛИНДРИЧЕСКИМИ ОБОЛОЧКАМИ
}

\author{
(Представил Н. Алумяэ)
}

Представляются результаты расчета частотной и временной зависимостей акустического давления, рассеянного оболочкой в жидкость. Проводится анализ временных зависимостей при резонансных и антирезонансных частотах возбуждения короткими и длинными волновыми пакетами. Выявляется природа формирования давления в рассеянном поле, находятся его основные компоненты и устанавливаются связи между ними. Показывается, что резонансы частотной зависимости имеют место, когда на длине окружности оболочки укладывается целое число длин волн некоторой нормальной (типа Лэмба) моды.

\section{1. Введение}

Проблема рассеяния акустических волновых пакетов цилиндрическими оболочками в жидкости интенсивно исследуется со времени экспериментального наблюдения переизлученных оболочкой волновых пакетов $\left[{ }^{1-3}\right]$.

В случае толстостенной оболочки наибольшие по амплитуде переизлученные волновые пакеты обусловлены распространяющейся по оболочке и непрерывно излучающей в жидкость упругой поверхностной волной типа Рэлея. Аналитически они были проанализированы в работах X. Юбералла с сотрудниками $\left[{ }^{4-6}\right]$. К решению задачи рассеяния в виде ряда по собственным функциям было применено интегральное преобразование Зоммерфельда-Ватсона и выполнен анализ особенностей подынтегральной функции, порождающих различные типы волн, бегущих по оболочке и непрерывно излучающих в жидкость. Местоположение полюсов на комплексной плоскости позволяет для каждого типа волн дать групповую и фазовую скорости, коэффициент затухания и критический угол возбуждения. В течение долгого времени информация о переизлученных волновых пакетах и ограничивалась названными величинами. Лишь недавно $\left[{ }^{7}\right]$ с помощью преобразования ЗоммерфельдаВатсона удалось провести вычисление вычетов в полюсах и построить частотную зависимость. Временная зависимость пока не сосчитана.

Экспериментальные исследования $\left[{ }^{8-17}\right]$ позволили качественно подтвердить механизм процесса рассеяния и определить групповые и фазовые скорости волн различных мод при некоторых фиксированных частотах (или в некоторых диапазонах частот). Коэффициенты затухания определялись с большой погрешностью. 
Стало уже традицией для решения задачи о рассеянии волн цилиндрической оболочкой использовать интегральное преобразование (Лапласа или Фурье) по времени с последующим применением метода разделения переменных и представлением решения в виде ряда по собственным функциям. В зависимости от степени сложности подынтегральной функции, а также умения и изобретательности автора обратное преобразование выполняется с различной степенью точности; при его проведении используются аналитические или численные методы.

Бытует мнение, что решение в виде ряда по собственным функциям (несмотря на то, что оно совершенно точное) неприемлемо, поскольку оно не прослеживает процесс рассеяния по компонентам, плохо приспособлено для описания разрывов, не работает в области высоких частот. Из этих соображений справедливо лишь первое. Для выделения разрывов решение надо расчленять на разрывную и гладкую части, как это предложил А. Н. Крылов; разрывную часть следует исследовать аналитически, а гладкую - раскладывать в ряд. Чтобы процесс вычисления решения в виде ряда по собственным функциям работал и при больших частотах, требуется искусство исследователя; об этом, в частности, свидетельствуют результаты $\left[{ }^{18}\right]$, полученные при изучении рассеяния акустических волн сплошными упругими цилиндрами и сферами при $x=900-950$ (здесь $x=k a-$ волновой радиус в жидкости). Во всяком случае в большей части имеющихся к настоящему времени работ по рассеянию на упругих телах расчеты выполнены путем непосредственного суммирования ряда по собственным функциям без привлечения интегрального преобразования Зоммерфельда-Ватсона.

При рассеянии на сплошном упругом цилиндре основной вклад в рассеянное поле вносят пакеты, порождаемые упругими поверхностными волнами типа Рэлея, обегающими оболочку по ее наружной и внутренней поверхностям. При переходе к тонким оболочкам основными составляющими рассеянного поля становятся уже нормальные (типа Лэмба) волны, которые формируются за счет суперпозиции волн типа Рэлея. Обусловленные этими нормальными волнами переизлученные волновые пакеты экспериментально исследовались довольно интенсивно $\left[{ }^{12}, 15,19\right]$, а аналитически весьма скромно - движение оболочки моделировалось лишь уравнениями теории тонких упругих оболочек $\left[{ }^{20-22}\right]$.

\section{2. Постановка задачи и метод решения}

В безграничной идеальной сжимаемой жидкости имеется пустая упругая цилиндрическая оболочка бесконечной протяженности. На нее набегает акустический волновой пакет давления, плоский фронт которого параллелен продольной оси оболочки (рис. 1). Изменение давления $p_{i}$ за фронтом волнового пакета может быть назначено произвольно

$$
\begin{gathered}
p_{i}(r, \theta, t)=p \cdot f(\zeta)[H(\zeta)-H(\zeta-\tau *)], \\
\zeta=t-(1-r \cos \theta), \quad H(\zeta)= \begin{cases}1 & \text { при } \zeta>0, \\
0 & \text { при } \zeta \leqslant 0,\end{cases}
\end{gathered}
$$

где $r, \theta-$ безразмерные радиальная и угловая координаты, $t-$ безразмерное время, $f$ - заданная функция, $\tau$. - длительность волнового пакета, p. - постоянная, имеющая размерность давления.

В формуле (1) и ниже используются следующие переменные 


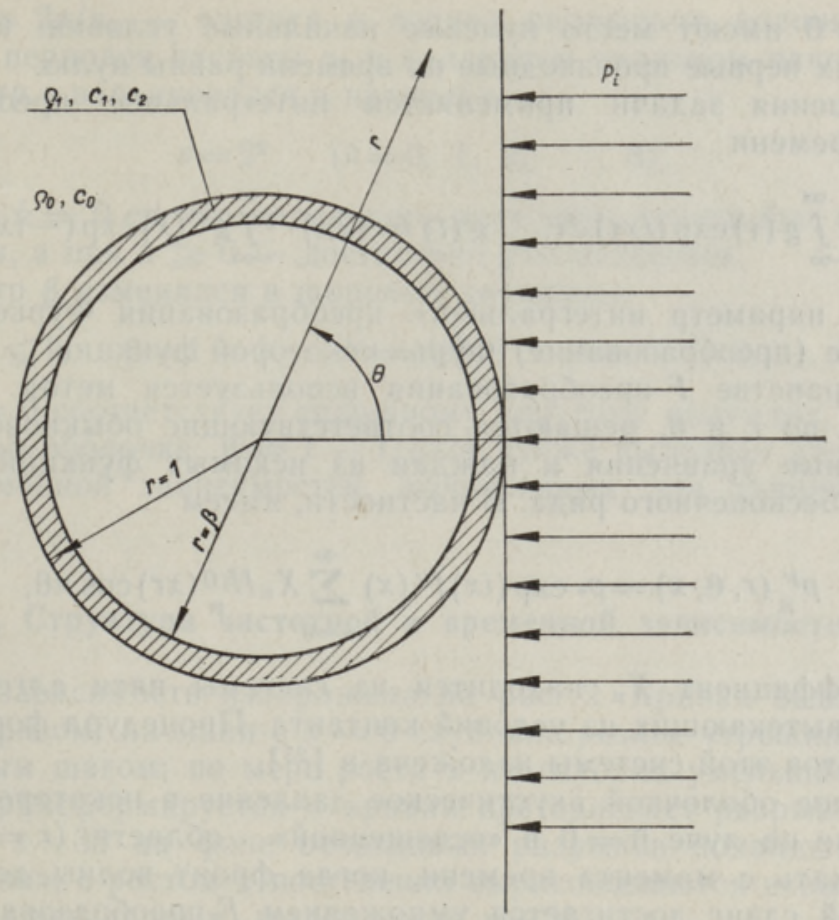

Рис. 1.

$$
r=R / a, \quad t=c_{0} T / a, \quad \beta=b / a,
$$

где $R$ - радиальная координата, $T$ - время, $a, b-$ наружный и внутренний радиусы оболочки, $c_{0}-$ скорость звука в жидкости. Время $t$ отсчитывается с момента, когда фронт падающего волнового пакета коснется поверхности оболочки в точке с координатами $r=1, \theta=0$. За единицу безразмерного времени $t$ фронт падающего волнового пакета проходит расстояние, равное наружному радиусу оболочки.

Рассеянное оболочкой поле давления $p_{S}$ удовлетворяет волновому уравнению

$$
\left(\nabla^{2}-\frac{\partial^{2}}{\partial t^{2}}\right) p_{S}=0, \quad r \geqslant 1
$$

где $\nabla^{2}-$ гармонический оператор.

На наружной поверхности оболочки при $r=1$ выполняются три условия контакта: непрерывность радиальных перемещений и напряжений, отсутствие тангенциальных напряжений; на внутренней поверхности оболочки при $r=\beta$ имеют место два условия: отсутствие радиальных и тангенциальных напряжений.

Движение оболочки определяется уравнениями линейной теории упругости, выписанными относительно потенциалов перемещений.

При $r \rightarrow \infty$ выполняется условие излучения

$$
\lim _{r \rightarrow \infty} r^{1 / 2}\left(\frac{\partial}{\partial r}+\frac{\partial}{\partial t}\right) p_{S}=0 .
$$

Все искомые функции полагаются ограниченными в областях, где они определены. 
При $t=0$ имеют место нулевые начальные условия: неизвестные функции и их первые производные по времени равны нулю.

Для решения задачи применяется интегральное преобразование Фурье по времени

$$
g^{F}(x)=\int_{-\infty}^{\infty} g(\tau) \exp (i x \tau) d \tau, \quad g(t)=(2 \pi)^{-1} \int_{-\infty}^{\infty} g^{F}(x) \exp (-i x t) d x .
$$

Здесь $x$ - параметр интегрального преобразования Фурье, $g^{F}(x)$ изображение (преобразование) Фурье некоторой функции.

В пространстве $F$-преобразования используется метод разделения переменных по $r$ и $\theta$, решаются соответствующие обыкновенные дифференциальные уравнения и каждая из искомых функций записывается в виде бесконечного ряда. В частности, имеем

$$
p_{S}^{F}(r, \theta, x)=p \cdot \exp (i x) F^{F}(x) \sum_{n=0}^{\infty} X_{n} H_{n}^{(1)}(x r) \cos n \theta,
$$

причем коэффициент $X_{n}$ находится из системы пяти алгебраических уравнений, вытекающих из условий контакта. Процедура формирования коэффициентов этой системы изложена в $\left[{ }^{23}\right]$.

Рассеянное оболочкой акустическое давление в некоторой фиксированной точке на луче $\theta=0$ в «освещенной» области $\left(r=r_{*}, \theta=0\right)$ удобно изучать с момента времени, когда фронт волны дойдет в эту точку. Такой сдвиг достигается умножением $F$-преобразования $(6)$ на $\exp \left[i x\left(r_{*}-2\right)\right]$. Тогда на луче $\theta=0 F$-преобразование акустического давления принимает вид

$$
p_{S}^{F}\left(r_{*}, 0, x\right)=p_{*} \exp \left[i x\left(r_{*}-1\right)\right] f^{F}(x) \sum_{n=0}^{\infty} X_{n} H_{n}^{(1)}\left(x r_{*}\right) .
$$

Применяя (5), вычислим оригинал этой функции

$$
\begin{gathered}
p_{S}\left(r_{*}, 0, t\right)=p \cdot(2 \pi)^{-1} \int_{-\infty}^{\infty}\left[\exp (i x \tau) f^{F}(x) \sum_{n=0}^{\infty} X_{n} H_{n}^{(1)}\left(x r_{*}\right)\right] d x, \\
\tau=t-\left(r_{*}-1\right) .
\end{gathered}
$$

Численный способ нахождения интеграла (8) описан в $\left[{ }^{23}\right]$.

Для удобсгва представления результатов пронормируем величины $(7),(8)$ на геометрический множитель и введем обозначения

$p^{F}(x)=p_{S}^{F}(x) / m F^{F}(x), \quad p(\tau)=p_{S}\left(r_{*}, 0, t\right) / m, \quad m=p_{*}\left(2 r_{*}-1\right)^{-1 / 2}$.

Ради краткости будем называть модуль функции $p^{F}(x)$ частотной зависимостью, а $p(\tau)$ - временной зависимостью.

Расчеты выполнялись для точки наблюдения $r .=10^{4}, \theta=0$ в случае размещения в воде стальной оболочки при следующих параметрах жидкой и упругой сред:

$$
\begin{array}{ll}
\mathrm{\varrho}_{0}=1,000 \mathrm{r} / \mathrm{CM}^{3}, & c_{0}=1493 \mathrm{M} / \mathrm{c}, \\
\mathrm{\varrho}_{1}=7,700 \mathrm{r} / \mathrm{CM}^{3}, & c_{1}=5960 \mathrm{M} / \mathrm{c}, \quad c_{2}=3240 \mathrm{M} / \mathrm{C}
\end{array}
$$

и синусоидальном падающем волновом пакете конечной длительности

$$
f(\zeta)=\sin x_{0} \zeta, \quad \tau_{*}=s \tau_{0},
$$


где $x_{0}, \tau_{0}=2 \pi / x_{0}$ - частота и период падающего волнового пакета, $s$ - число периодов частоты $x_{0}$ в падающем волновом пакете.

Параметр $s$ варьировался в пределах

$$
s=2^{k} \quad(k=0,1,2, \ldots, 8),
$$

причем при $k=0$ спектр падающего волнового пакета был очень широкополосным, а при $k \geqslant 6-$ достаточно узкополосным.

Параметр $\beta$ изменялся в широком диапазоне

$$
0 \leqslant \beta \leqslant 1, \quad \beta=1-h, \quad h=(1 / 2)^{k} \quad(k=0,1,2, \ldots, 10) .
$$

Случай $k=0$ соответствует сплошному упругому цилиндру, а $k=10-$ очень тонкой оболочке: $\beta=1-1 / 1024$. Ниже выполнен анализ частотной и временной зависимостей, вычисленных для типичного случая $h=1 / 512$.

\section{3. Структура частотной и временной зависимостей}

Частотная зависимость изображена на рис. 2. Кривая ведет себя следующим образом: начиная с $x=0$ глубокие резкие «трещины» следуют с постоянным шагом; по мере роста $x$ их глубина уменьшается, форма медленно трансформируется и кривая претерпевает разрыв при $x \sim 30$. Начиная с $x \sim 35$ на фоне отчетливых разрывов появляются небольшие «складки», с ростом $x$ постепенно вырисовываются невысокие пики, следующие с регулярным шагом после $x \sim 50$.

Такое поведение кривой $|p|$ легко объясняется наложением двух преобладающих мод, одна из которых - безмоментная - отчетливо
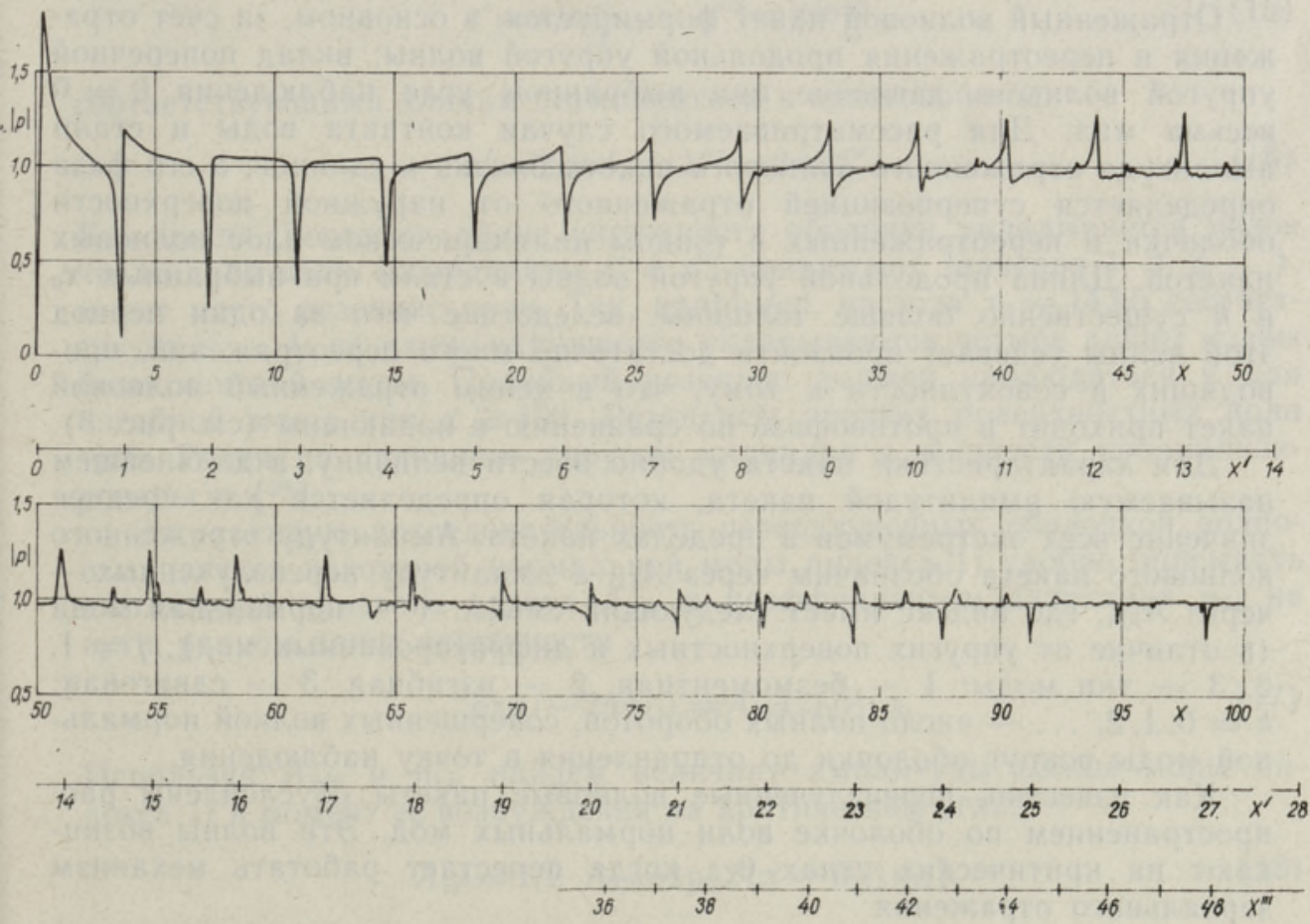

Рис. 2. 


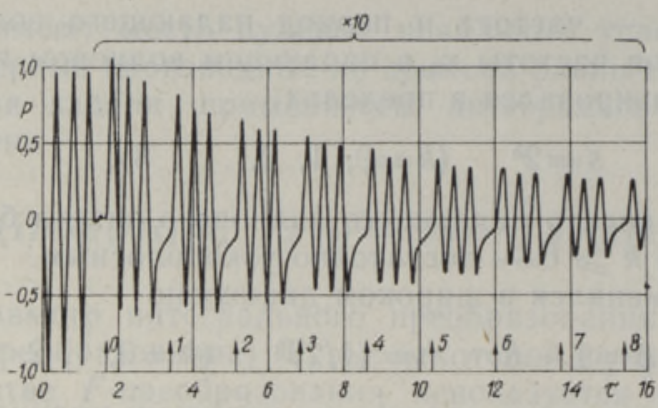

Рис. 3.

различается во всем рассматриваемом диапазоне частот, а другая сдвиговая - только при $x=75-95$.

Отметим, что в случае более толстостенных оболочек в некотором узком диапазоне частот наблюдается и изгибная мода. Здесь при $h=1 / 512$ она проявляется при $x \geqslant 400$.

На рис. 3 показана временная зависимость, рассчитанная при $x_{0}=14,65, s=3$. Один падающий волновой пакет вызывает последовательность пакетов в рассеянном поле, первый из которых (в дальнейшем называемый отраженным) обусловлен отражением от наружной поверхности оболочки и переотражением в тонком цилиндрическом слое, а последующие, в основном, - переизлучением. При частоте $x_{0}=14,65$ вкладом дифрагированных волновых пакетов в акустическое поле можно пренебречь ввиду их малости.

Для улучшения обозримости результатов вычислений масштаб переизлученных оболочкой волновых пакетов выбран в десять раз большим, чем масштаб отраженного.

Отраженный волновой пакет формируется, в основном, за счет отражения и переотражения продольной упругой волны; вклад поперечной упругой волны в давление при выбранном угле наблюдения $\theta=0$ весьма мал. Для рассматриваемого случая контакта воды и стали амплитуда отраженного волнового пакета близка к единице, а его фаза определяется суперпозицией отраженного от наружной поверхности оболочки и переотраженных в тонком цилиндрическом слое волновых пакетов. Длина продольной упругой волны в стали при выбранных $x_{0}$ и $h$ существенно больше толщины, вследствие чего за один период этой волны успевает произойти достаточно много переотражений, приводящих в совокупности к тому, что в целом отраженный волновой пакет приходит в противофазе по сравнению с падающим (см. рис. 3).

Для характеристики пакета удобно ввести величину, в дальнейшем называемую амплитудой пакета, которая определяется как среднее значение всех экстремумов в пределах пакета. Амплитуду отраженного волнового пакета обозначим через $A_{11}$, а амплитуду переизлученных через $A_{1 j k}$, где индекс имеет следующий смысл: 1 - нормальная мода (в отличие от упругих поверхностных и дифрагированных мод); $j=1$, 2,3 - тип моды: 1 - безмоментная, 2 - изгибная, 3 - сдвиговая; $k=0,1,2, \ldots$ - число полных оборотов, совершенных волной нормальной моды вокруг оболочки до отправления в точку наблюдения.

Қак известно, переизлученные волновые пакеты обусловлены распространением по оболочке волн нормальных мод. Эти волны возникают на критических углах $\theta_{1 j}$, когда перестает работать механизм зеркального отражения

$$
\theta_{1 j}=\arcsin \left[\left(v_{1 j}^{p h}\right)^{-1}\right], \quad v_{1 j}^{p h}=c_{1 j}^{p h} / c_{0},
$$


где $c_{1 j}{ }^{p h}-$ фазовая скорость волны $j$-й нормальной моды.

Волны нормальных мод, обегая оболочку по часовой стрелке и против нее, непрерывно излучают в жидкость. В процессе распространения их амплитуда падает, вследствие чего уменьшаются и амплитуды последовательных переизлученных пакетов. Если точка наблюдения находится на лучах $\theta=0$ и $\theta=\pi$, последние приходят в эту точку одновременно и суммируются в фазе.

Используя разность времен прихода некоторой характерной точки (максимума, минимума, нуля) $(k+1)$-го и $k$-го переизлученных волновых пакетов, найдем фазовую скорость волны нормальной моды. При $x_{0}=14,65$ получим $v_{11}^{p h}=3,66$.

Хотя падающий волновой пакет при $s=3$ имеет широкий спектр, в интеграле (8) он перекрывает полосу, описываемую одной единственной безмоментной нормальной модой.

Как известно $\left[{ }^{5}\right]$, интервал между двумя последовательными сходственными точками частотной зависимости (в конкретной ситуации между характерными минимумами) определяет относительную (разделенную на $c_{0}$ ) групповую скорость волны некоторой нормальной моды (в данном случае - безмоментной).

Во всем рассматриваемом диапазоне частот $x=0-100$ эта мода распространяется практически без дисперсии - шаг между характерными минимумами не изменяется и равняется 3,66 . Равенство групповой и фазовой скоростей также указывает на ее бездисперсионность.

По частотной зависимости (см. рис. 2) легко определяется и групповая скорость волны сдвиговой моды $v_{13}{ }^{p h}=2,06$. В диапазоне частот, где эта мода отчетливо видна, дисперсия скорости отсутствует и у нее.

Мы снабдили рис. 2 двумя дополнительными осями

$$
x^{\prime}=x / v_{11}^{p h}, \quad x^{\prime \prime \prime}=x / v_{13}^{p h},
$$

соответствующими длинам безмоментной и сдвиговой волн

$$
\lambda^{\prime}=2 \pi / x^{\prime}, \quad \lambda^{\prime \prime \prime}=2 \pi / x^{\prime \prime \prime} .
$$

Как видно, когда на длине окружности оболочки укладывается целое число длин волн (координаты $x^{\prime}$ и $x^{\prime \prime \prime}$ принимают значения $1,2,3, \ldots$ ), имеет место резонанс волн. Так, например, частота $x_{0}=14,65$ соответствует $x^{\prime}=4$ : на длине окружности укладываются четыре длины волны безмоментной моды. Подобный резонанс должен наблюдаться и для изгибной моды при $x \geqslant 400$. Резонансы упругих поверхностных волн типа Рэлея на сплошном упругом цилиндре в жидкости проанализированы в $\left[{ }^{24}\right]$.

Затухающую последовательность переизлученных оболочкой волновых пакетов некоторой нормальной моды индекса $1 j$ удобно описывать амплитудой нулевого пакета $A_{1 j 0}$ и коэффициентом затухания $\eta_{1 j}$ на пути, равном длине окружности

$$
\exp \left(-2 \pi \eta_{1 j}\right)=A_{1 j(k+1)} / A_{1 j k} .
$$

Используя $A_{1 j 0}$ и $\eta_{1 j}$, найдем величину амплитуды волны моды индекса $1 j$ в момент ее возбуждения на критическом угле:

$$
A_{1 j^{*}}=1 / 2 \quad A_{1 j 0} \exp \left[2\left(\pi-\theta_{1 j}\right) \eta_{1 j}\right] .
$$

При выбранных параметрах задачи все величины, характеризующие рассеянное оболочкой давление, сведены в таблицу. 


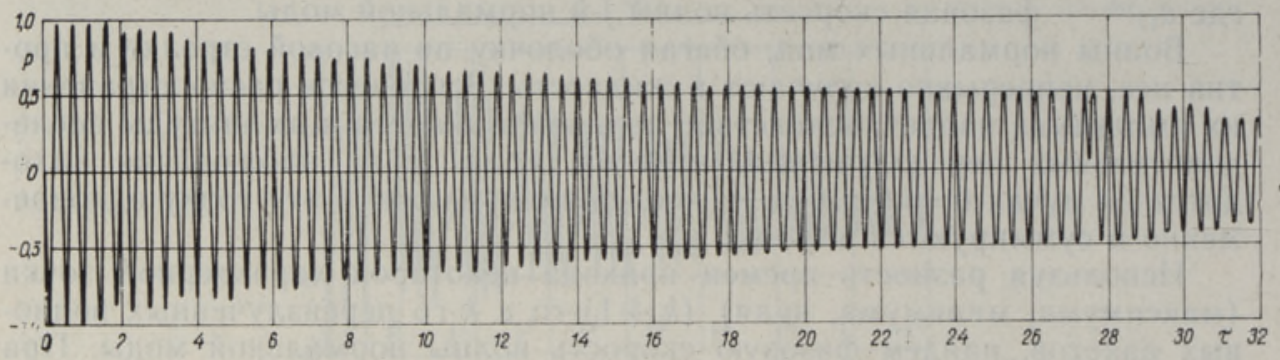

Рис. 4.

Параметры волновых пакетов безмоментной моды при резонансном и антирезонансном рассеянии

\begin{tabular}{c|c|c|c|c|c}
\hline$x_{0}$ & 10,97 & \multicolumn{2}{|c|}{12,81} & \multicolumn{2}{|c}{14,65} \\
\cline { 2 - 5 }$x^{\prime}$ & 3 & \multicolumn{2}{|c|}{3,5} & \multicolumn{2}{|c}{4} \\
\hline$s$ & 64 & 3 & 64 & 3 & 64 \\
$A_{11}$ & 0,978 & 0,985 & 0,984 & 0,985 & 0,988 \\
$B_{11}$ & 0,375 & - & 1,032 & - & 0,502 \\
$B_{11 *}$ & 0,371 & - & 1,033 & - & 0,477 \\
$C_{11}$ & 0,614 & - & - & - & 0,490 \\
$A_{110}$ & 0,092 & 0,088 & - & 0,081 & 0,072 \\
$A_{11 *}$ & 0,054 & 0,051 & - & 0,047 & 0,041 \\
$\eta_{11}$ & 0,027 & 0,025 & - & 0,026 & 0,024
\end{tabular}

Введем параметр $\tau_{1 j}$, задающий время, необходимое волне некоторой нормальной моды на совершение одного полного оборота вокруг оболочки

$$
\tau_{1 j}=2 \pi / v_{1 j}^{p h} .
$$

Для безмоментной моды $\tau_{11}=1,72$; при выбранном $s=3$ величина $\tau *=1,29<\tau_{11}$ и в силу этого переизлученные волновые пакеты, порождаемые волной безмоментной моды, приходят порознь, не накладываясь друг на друга.

На рис. 4 изображена временная зависимость, рассчитанная при $x_{0}=14,65, s:=64$. Теперь $\tau \cdot=27,45>\tau_{11}$ и поэтому переизлученные волновые пакеты накладываются друг на друга. Времена прихода, амплитуды и фазы последних схематически показаны на рис. 5.

Рассеянное оболочкой давление обусловлено, как и ранее, процессами отражения и переотражения. На отраженный волновой пакет (не показанный на рис. 5) последовательно накладываются переизлученные волновые пакеты. Как видно из рис. 4, фазы их не совпадают $A_{11} \gg A_{110}$, поскольку амплитуда суперпозиции отраженного и нулевого переизлученного волновых піакетов мало отличается от амплитуды $A_{11}$. Последовательно переизлученные волновые пакеты приходят с постоянным шагом $x^{\prime}=4$, причем каждый раз с меньшей амплитудой («ступеньки» становятся все ниже), в связи с чем величина амплитуды суперпозиции постепенно уменьшается и в конце концов выходит на квазиасимптотический уровень. Поскольку расчет выполняется с конечной точностью, за этот уровень можно принять то значение амплитуды суперпозиции, при котором величина неучтенного переизлученного волнового пакета меньше погрешности вычислений.

Асимптотическое значение $B_{11}$. достигается при $s \rightarrow \infty$. Это значение 


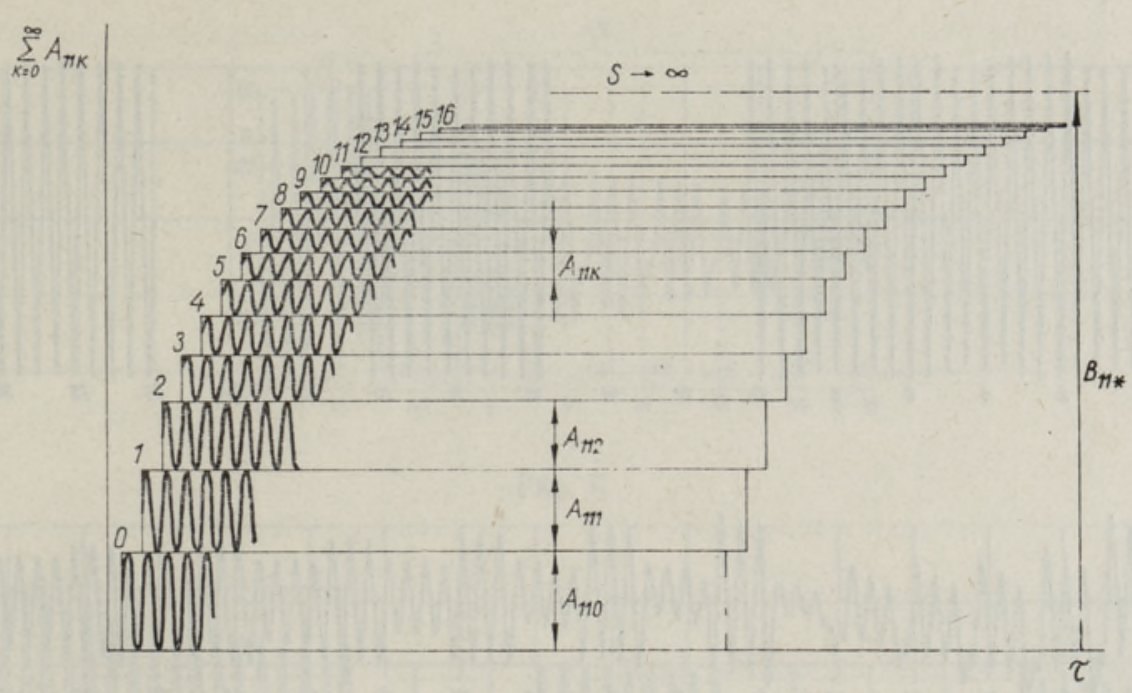

Рис. 5.

соответствует величине $|p|$ при $x=x_{0}$ на частотной зависимости (см. рис. 2).

При $\tau .>\tau$. (рис. 4) переизлученные волновые пакеты можно наблюдать в чистом виде, а не в суперпозиции с отраженным, как это было при $\tau<\tau$. Сперва видно начало волнового пакета, сформировавшегося в результате суперпозиции всех переизлученных пакетов. Амплитуду этой суперпозиции мы обозначим через $C_{11}$. За ним со сдвигом по $\tau$ на $\tau_{11}$ следуют начала волновых пакетов, образованных суперпозицией всех переизлученных пакетов без нулевого, без нулевого и первого, без нулевого, первого и второго и т. д. Амплитуду каждого начала (усредненную величину всех экстремумов) обозначим через $C_{110}$, $C_{111}, C_{112}, C_{113}, \ldots$. Располагая их значениями, можно легко определить амплитуды последовательных переизлученных волновых пакетов, обусловленных безмоментной модой, и коэффициент затухания

$$
\begin{aligned}
A_{110}= & C_{11}-C_{110}, \quad A_{11 k}=C_{11 k}-C_{11(k+1)}, \\
& \exp \left(-2 \pi \eta_{11}\right)=A_{11(k+1)} / A_{11 k} .
\end{aligned}
$$

Как и прежде, используя $A_{110}$ и $\eta_{11}$, легко найти $A_{11}$. Числовые значения, относящиеся к рассматриваемому примеру, приведены в таблице.

Сопоставление двух колонок таблицы, соответствующих длинному и короткому падающим волновым пакетам $(s=3$ и $s=64)$, показывает, что амплитуды начал отраженных волновых пакетов, как и следовало ожидать, не зависят от протяженности падающего. Амплитуда $A_{11}$. при $s=3$ несколько больше, чем при $s=64$ в силу того, что спектр падающего волнового пакета в последнем случае значительно ужже спектра при $s=3$. Коэффициенты затухания, вычисленные при $s=3$ и $s=64$, немного различаются между собой, что можно объяснить погрешностью вычислений. Действительно, если при $s=64$ для нахождения $\eta_{11}$ использовать формулу убывающей геометрической прогрессии

$$
\exp \left(-2 \pi \eta_{11}\right)=1-A_{110} / C_{11}
$$



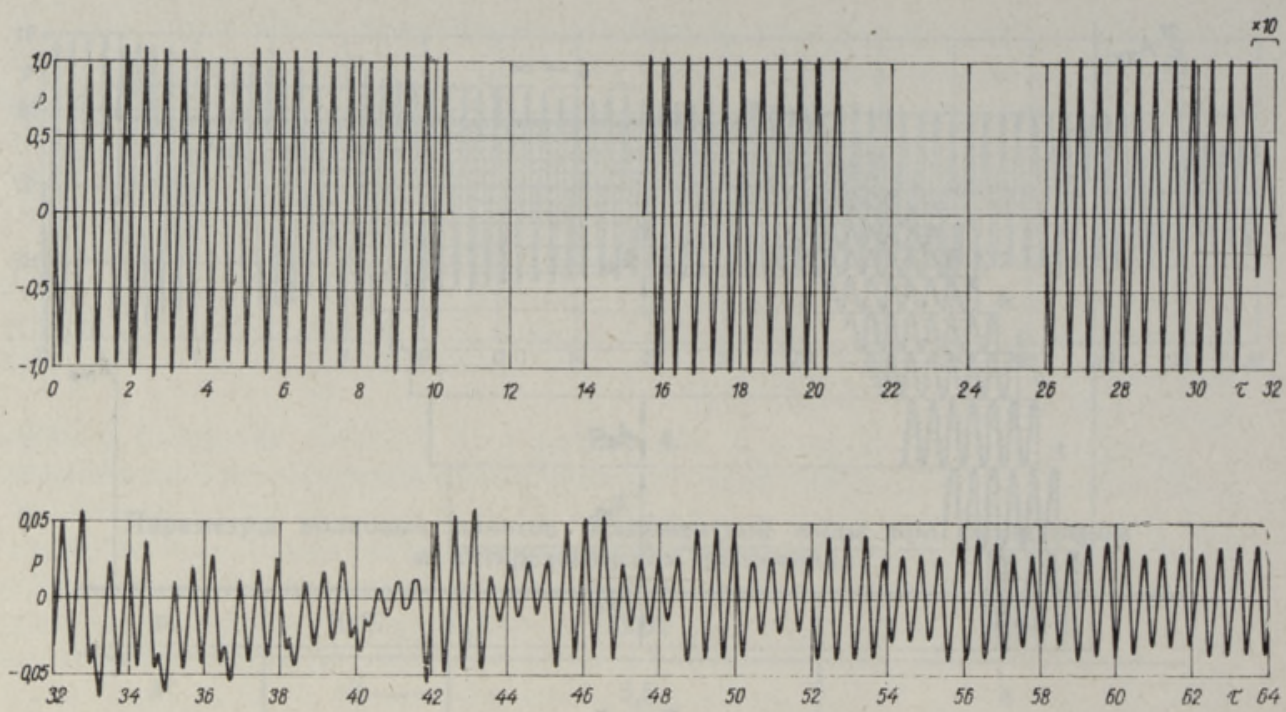

Рис. 6.

то получим значение $\eta_{11}=0,025$, лежащее между значениями этого коэффициента, приведенными в таблице.

Если частота падающего волнового пакета соответствует антирезонансу нормальных волн (на двух длинах окружности укладывается целое число длин нормальных волн), то последние, обегая оболочку, будут накладываться на себя в фазе через два, четыре, шесть и т. д. оборотов и в противофазе - через один, три, пять и т. д. оборотов. В результате этого при четном числе оборотов происходит усиливающаяся интерференция, а при нечетном - ослабевающая. Это демонстрирует рис. 6, где отражен результат расчета при $x_{0}=12,81$ и $s=64$. Выбранная частота $x_{0}$ соответствует $x^{\prime}=3,5$, так что через семь периодов падающей волны кривая $p(\tau)$ должна принять сходственную с первоначальной форму. Из рис. 6 видно, что после начала отраженного волнового пакета выстраивается характерная «лестница», причем максимумы следуют «ступеньками» шириной 4-3-4-3- . . , а минимумы шириной 3-4-3-4- ... периода падающего волнового пакета. Высоты «ступенек» выравниваются с ростом $\tau$, поскольку вклад в давление каждого переизлученного волнового пакета убывает с ростом индекса $k$, а амплитуда огибающей кривой $p(\tau)$ выходит на квазиасимптотический уровень $B_{11}$. Любопытно отметить, что в данном примере квазиасимптотический уровень весьма близок к асимптотическому, определяемому по частотной зависимости, где $s \rightarrow \infty$.

При выбранной частоте $x_{0}=12,81$ фазы отраженного и всех переизлученных волновых пакетов близки и поэтому амплитуда суперпозиции этих пакетов больше амплитуды отраженного.

По окончании падающего волнового пакета процесс отражения прекращается, а переизлучения - продолжается. При $\tau>\tau$. отчетливо видны области усиливающейся и ослабевающей интерференции (отметим, что масштаб кривой $p(\tau)$ при $\tau>\tau$. в десять раз больше, чем при $\tau<\tau *)$. Здесь также наблюдается характерная «лестница»: максимумы кривой $p(\tau)$ идут ступеньками шириной 4-3-4-3- ..., а минимумы - 


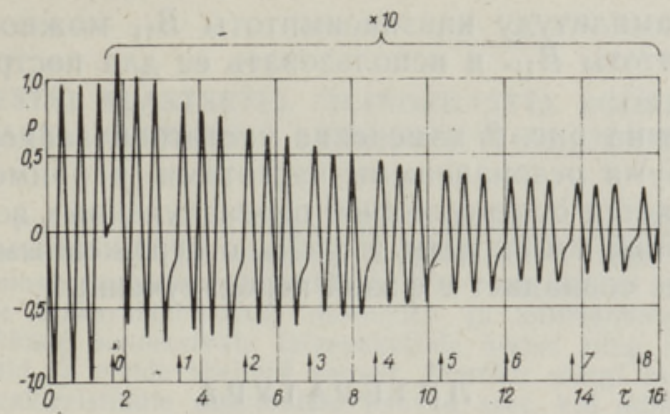

Рис. 7.
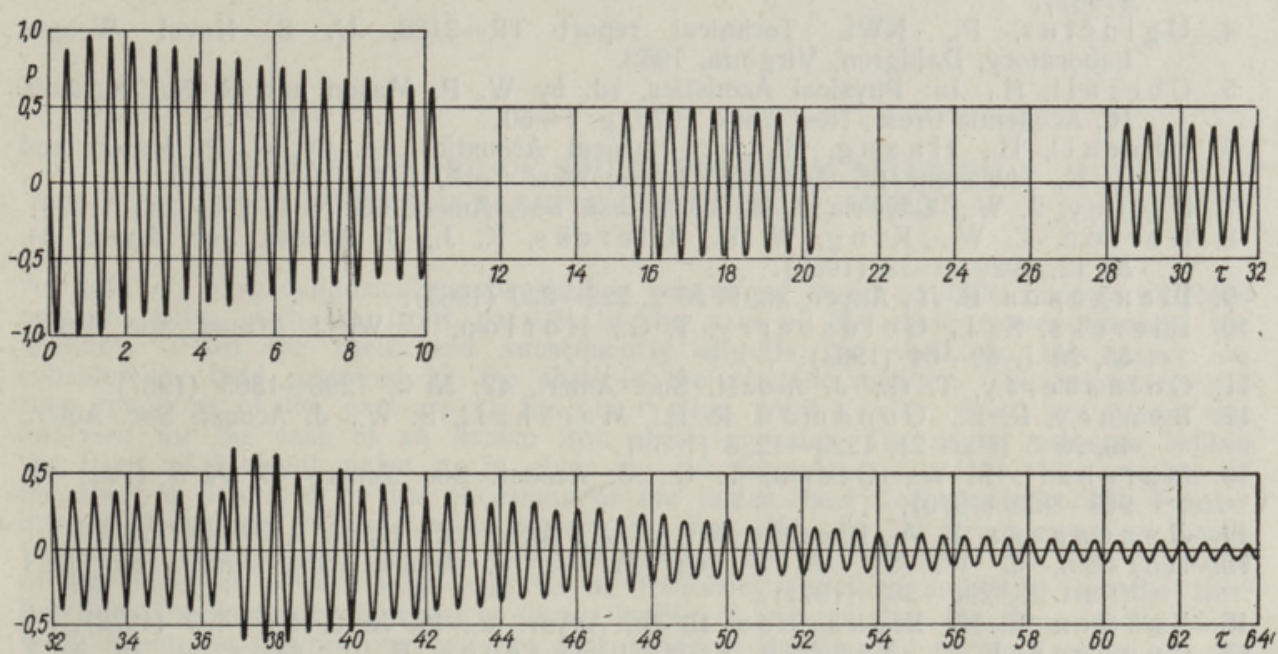

Рис. 8.

шириной 3-4-3-4- ... периода падающего волнового пакета. По мере роста $\tau$ высота ступенек уменьшается.

На рис. 7 и 8 показаны временные зависимости, рассчитанные при $x_{0}=12,81, s=3$ и $x_{0}=10,97, s=64$ соответственно; параметры волновых пакетов приведены в таблице. Качественно процесс рассеяния идет здесь так же, как и в подробно описанных выше случаях.

Из сопоставления различных столбцов таблицы видно, что амплитуда нулевого переизлученного волнового пакета $A_{110}$ падает с ростом частоты $x_{0}$, а коэффициент затухания $\eta_{11}$ практически не изменяется. Этим обстоятельством объясняется уменьшение глубины «трещины» на частотной зависимости при переходе от одной резонансной частоты к соседней.

Руководствуясь амплитудой $A_{110}$ и коэффициентом затухания $\eta_{11}$ и задавая некоторую погрешность измерения $\varepsilon$, можно подсчитать число периодов в падающем волновом пакете, при котором амплитуда огибающей $p(\tau)$ выходит на квазиасимптотический уровень:

$$
s=\ln \left(A_{110} / \varepsilon\right) / 2 \pi \eta_{11} .
$$

5 ENSV TA Toimetised. F*M 21981 
В этом случае амплитуду квазиасимптоты $B_{11}$ можно считать равной амплитуде асимптоты $B_{11}$. и использовать ее для построения частотной зависимости.

Наблюдаемое на рис. 2 изменение частотной зависимости в промежутке между двумя резонансными частотами, например между $x^{\prime}=4$ и $x^{\prime}=5$, объясняется суперпозицией переизлученных волновых пакетов, имеющих различные амплитуды и фазы, с отраженным, фаза которого, вообще говоря, не совпадает с фазой переизлученных.

\section{ЛИТЕРАТ У РА}

1. Л я м ш в В. М., Акуст. ж., 2, № 2, 188-193 (1956).

2. К уз м ичев Ю. М., М ак а ров В. И., Акуст. ж., 4, № 3, $282-283$ (1958).

3. B a rn a rd, G. R., M K K inne y, C. M., J. Acoust. Soc. Amer., 33, № 2, 226-238 (1961),

4. Uginčius, P., NWL Technical report TR-2128, U. S. Naval Weapos Laboratory, Dahlgren, Virginia, 1968.

5. Ubera 11, H., In: Physical Acoustics, ed. by W. P. Mason and R. N. Thurston, 10, Academic Press, New York, 1973, p. 1-60.

6. Uberall, H., $\mathrm{Hu}$ a ng, H., In: Physical Acoustics, ed. by W. P. Mason and R. N. Thurston, 12, Academic Press, New York, 1976, p. 217-275.

7. D ickey, J. W., Úber a 11, H., J. Acoust. Soc. Amer., 66, № 1, 275-283 (1979),

8. Horton, C. W., King, W. R., Diercks, K. J., J. Acoust. Soc. Amer., 34, № 12, 1929-1932 (1962).

9. Ш енде р о в Е. Л., Акуст. ж., 9, № 2, $222-230$ (1963).

10. Diercks, K. J., Goldsberry, T. G., Horton, C. W., J. Acoust. Soc. Amer., 35, № 1, 59-64 (1963).

11. Goldsberry, T. G., J. Acoust. Soc. Amer., 42, № 6, $1298-1305$ (1967).

12. Bunney, R. E., Goodma n, R. R., M a r sha 11, S. W., J. Acoust. Soc. Amer., 46, № 5 (Part 2), 1223-1233 (1969).

13. Marsha11, S. W., O 1 so n, T. G., J. Acoust. Soc. Amer., 47, № 3 (Part 2), $949-950(1970)$

14. Лихода ева Е. А., Ш ендеров Е. Л., Акуст. ж., 17, № 1, $79-84$ (1971).

15. Horton, C. W. Sr., Mechler, M. V., J. Acoust. Soc. Amer., 51, № 1 (Part 2), 295-303 (1972).

16. Х ей фец Е. И., Ш е де ров Е. Л., Акуст. ж., 18, № 3, 456-463 (1972).

17. С ми нов Е. П., Хей фец Е. И., Шендеров Е. Л., Акуст. ж., 19, № 2, $240-250$ (1973).

18. F 1 a x, L., J. Acoust. Soc. Amer., 62, № 6, 1502-1503 (1977).

19. R y a n, W. W. Jr., J. Acoust. Soc. Amer., 64, № 4, 1159-1164 (1978),

20. Hua ng, H., W a n g, J. F., J. Acoust. Soc. Amer., 48, № 1, (Part 2), 228-235 $(1970)$.

21. T a n g, S. C., Ye h, D. H. Y., J. Acoust. Soc. Amer., 47, № 5, 1325-1333 (1970).

22. Метсавээр Я. А., В екслер Н. Д., С тулов А. С., Дифракция акустических импульсов на упругих телах, М., «Наука», 1979.

23. В екслер Н. Д., Рассеяние импульсов на упругих цилиндрах, Таллин, «Валгус», 1980.

24. Dickey, J. W., Uber a 11, H., J. Acoust. Soc. Amer., 63, № 2, 319-320 (1978).

Институт кибернетики

Академии наук Эстонской ССР
Поступила в редакцию $6 /$ I 1981 


\section{OHUKESTEL ELASTSETEL SILINDRILISTEL KOORIKUTEL HAJUVATE AKUSTILISTE LAINEPAKETTIDE OLEMUSEST}

Artiklis on esitatud vedelikus asuval väga õhukeseseinalisel koorikul hajunud akustilise rōhu arvutamise tulemused aja ja sageduse funktsioonidena. Kooriku liikumist on kirjeldatud lineaarse elastsusteooria vōrranditega. On analüüsitud momendivaba moodi poolt tekitatud laine ajakarakteristikuid resonants- ja antiresonantssagedustel niihästi lühikeste kui ka pikkade sondeerivate lainepakettide korral ning leitud hajunud välja põhilised komponendid ja nendevahelised seosed. Kooriku mingi moodi tekitatud lainepakettide sageduskarakteristikute resonantsid leiavad aset, kui mingi Lambi tüüpi normaalmoodi lainepikkus mahub kooriku perimeetrile täpselt täisarv kordi.

N. VEKSLER

\section{THE NATURE OF SOUND PULSES SCATTERING BY THIN ELASTIC CYLINDRICAL SHELLS}

An infinitely long cylindrical elastic shell is submerged in an acoustic medium. A plane pressure pulse, with the front parallel to the axis of the shell, moves through the medium, strikes the shell, and subsequently engulfs the shell. In this paper we consider the field scattered by the shell in the acoustic medium. The frequency and time functions of the pressure at the observation point $r=10^{4}, \Theta=0$ are given and analysed for the case of an Armco iron shell in water. The sound pressure behind the front of incident pulse $p_{i}$ is given by the formulae (1), (11). The motion of the shell is described by the equations of the linear theory of elasticity. The Fourier integral transform is used with respect to time (5). In the transformation space the sound pressure on the ray $\Theta=0$ is defined by the formulae (8). The modulus of the function $p^{F}(x)$ is referred to as frequency function and $p(\tau)$ as the time function. The frequency function is shown in Fig. 2 and the time functions in Fig. 3, 4, 6-8. From the figures one can see that at short duration of incident pulse, all the wave pulses re-radiated by the shell sound came separately (Fig. 3, 7); whereas at long duration of incident pulse, they overlap each other. The extrema of the frequency function occur when the shell circumference is exactly an integer number of some normal (Lamb type) wavelenghts. At this frequency the resonance takes place in the time function: the sound wave pulses re-radiated by the shell came in the same fase (Fig. 4). If the shell circumference is one half of an integer number of some normal wavelengths, the anti-resonance takes place in the time function (Fig. 6). 